\title{
Less adhesiolysis and hernia repair during completion proctocolectomy after laparoscopic emergency colectomy for ulcerative colitis
}

\author{
Sanne A. L. Bartels • Malaika S. Vlug • \\ Daan Henneman - Cyriel Y. Ponsioen • \\ Pieter J. Tanis · Willem A. Bemelman
}

Received: 17 February 2011/Accepted: 29 July 2011/Published online: 13 October 2011

(C) The Author(s) 2011. This article is published with open access at Springerlink.com

\begin{abstract}
Background The aim of this study was to determine whether the need for adhesiolysis during completion proctectomy $(\mathrm{CP})$ with ileopouch anal anastomosis (IPAA) is influenced by the surgical approach of the initial emergency colectomy for ulcerative colitis and the hospital setting.

Methods One hundred consecutive patients who underwent CP with IPAA in our center between January 1999 and April 2010 were included. Emergency colectomy had been performed laparoscopically in 30 of 52 patients at the Academic Medical Center Amsterdam and in 6 of 48 patients at referring hospitals. Case files of these patients were retrospectively reviewed.

Results Significantly more extensive adhesiolysis was performed after open compared to laparoscopic colectomy (47 vs. $6 \%, P<0.001$ ). In univariate analysis, emergency colectomy at a referring hospital was also predictive for adhesiolysis $(P=0.003)$, but the open approach for the initial colectomy was the only independent predictive factor for the need for adhesiolysis $(P<0.001)$ in a multivariable ordinal logistic regression analysis. Operating time of CP was significantly longer when limited [18 (95\% $\mathrm{CI}=0-36) \mathrm{min}$ ] or extensive [55 (35-75) $\mathrm{min}$ ] adhesiolysis had to be performed. The interval to $\mathrm{CP}$ was longer after open colectomy and after colectomy performed at a
\end{abstract}

S. A. L. Bartels - M. S. Vlug · D. Henneman ·

P. J. Tanis · W. A. Bemelman ( $₫)$

Department of Surgery, Academic Medical Center,

PO Box 22660, 1100 Amsterdam, The Netherlands

e-mail: w.a.bemelman@amc.uva.nl

C. Y. Ponsioen

Department of Gastroenterology \& Hepatology, Academic

Medical Center, Amsterdam, The Netherlands referring hospital. Significantly more incisional hernia corrections during $\mathrm{CP}$ were performed after open emergency colectomy (14 vs. $0 \%, P=0.024)$. Overall morbidity and postoperative hospital stay of $\mathrm{CP}$ were not related to the surgical approach or the hospital setting of the emergency colectomy.

Conclusion Laparoscopic as opposed to open emergency colectomy is associated with less adhesiolysis, fewer incisional hernias, and a shorter interval to completion proctectomy.

Keywords Restorative proctocolectomy . Ulcerative colitis - Abdominal adhesions . Laparoscopy · Incisional hernia

The incidence of ulcerative colitis (UC) is estimated to be 2-20 per 100,000 in Western countries and has been rising $[1,2]$. Up to $32 \%$ of UC patients undergo surgical treatment eventually [3, 4]. In the case of severe refractory UC and life-threatening symptoms (e.g., toxic megacolon, imminent perforation), an emergency colectomy is performed, leaving the rectal stump and an ileostomy in situ. After the patient has sufficiently recovered, a completion proctectomy (CP) with creation of an ileopouch anal anastomosis (IPAA) is carried out. Laparoscopy has been successfully used in many colorectal procedures over the last two decades, including emergency colectomy for UC and the IPAA [5]. Recent studies suggest a decrease in adhesion formation after laparoscopic colectomy in addition to its cosmetic advantages [6, 7]. Postoperative adhesions are associated with small-bowel obstruction, secondary infertility, and chronic abdominal pain [7]. Furthermore, the operating time of subsequent abdominal interventions is increased due to adhesiolysis, which can 
range from $15 \mathrm{~min}$ to $4 \mathrm{~h}$ [8]. In addition, these consequences of adhesions lead to a large financial burden [9]. Therefore, strategies to reduce adhesion formation are of clinical and economical importance. Another advantage of laparoscopy is the possible reduction in incisional hernias [10]. Incisional hernias occur in $5-11 \%$ of midline laparotomies, one third of which become symptomatic at some point in time [11]. CP with IPAA is performed mostly in academic centers by experienced gastrointestinal surgeons, while the emergency colectomy is not infrequently done by general surgeons without laparoscopic experience at referring hospitals.

At present, it is not clear if laparoscopic emergency colectomy leads to less adhesion formation or a reduction in incisional hernias. The two-stage nature of the emergency colectomy and the subsequent $\mathrm{CP}$ with IPAA creates the opportunity to record the need for adhesiolysis and incisional hernia correction during the second procedure. The aim of this retrospective study was to determine whether the need for adhesiolysis during $\mathrm{CP}$ and IPAA creation is influenced by the approach, i.e., open or laparoscopic, or the hospital of the initial colectomy, i.e., referring or academic hospital.

\section{Materials and methods}

\section{Patients}

All patients that underwent CP with IPAA for UC from January 1999 through April 2010 were identified from a prospective database of the Academic Medical Center (AMC), Amsterdam, The Netherlands. All CPs were done by or under the supervision of two colorectal surgeons. Data were retrieved from patient charts, operative notes, pathology reports, and the complication registry of both referring hospitals and the AMC. Postoperative outcomes were extracted, including postoperative length of stay in days and complications such as anastomotic leakage, abscesses, paralytic ileus, and surgical site infections. All referring hospitals were community hospitals, both teaching and nonteaching.

\section{Outcome measures}

Primary outcome measure was the extent of adhesiolysis during CP with IPAA. During CP with IPAA, the extent of adhesiolysis was routinely reported in the operative notes by the surgeon. Adhesiolysis was scored as "none," "limited," or "extensive" based on these notes. Secondary outcome measures were operating time, incisional hernia correction during $\mathrm{CP}$, morbidity, mortality, postoperative length of stay, and the time interval to $\mathrm{CP}$. A minor complication was defined as a complication that could be managed conservatively. Complications requiring any surgical or percutaneous intervention or an admission to the Intensive Care Unit were defined as a major complication, and overall morbidity was defined as any complication requiring a medical intervention within 30 days after the index operation. Conversion was defined as unplanned laparotomy or extension of the initial extraction site incision; patients who underwent conversion were analyzed as laparoscopic colectomy.

Primary and secondary outcome measures were compared for the open and laparoscopic approaches of the emergency colectomy and for whether the initial surgery was performed in a referring center or the AMC. To identify independent factors predictive of the need for adhesiolysis, operating time, and incisional hernia correction during $\mathrm{CP}$ with IPAA, potentially related variables were entered in a multivariable regression model.

\section{Surgical techniques}

The surgical approach of the emergency colectomy was at the discretion of the surgeon and depended on the laparoscopic expertise of the surgeon. Open colectomy was carried out through a midline incision. Laparoscopic colectomy was performed mostly hand-assisted with the aid of a hand port inserted into a Pfannenstiel incision. Four patients underwent total laparoscopic colectomy in which the resected specimen was extracted transrectally in two patients and via the future end ileostomy opening in two patients. CP with IPAA was carried out through a midline or a Pfannenstiel incision, depending on the approach of the initial colectomy. The small bowel mesentery was mobilized up to the duodenum in all patients in order to ensure optimal pouch reach. J-pouch technique was used for IPAA construction. In patients with an increased risk of anastomotic leakage, i.e., prednisone dose of more than $20 \mathrm{mg}$ or technical problems, a loop ileostomy was created for the protection of the IPAA.

\section{Statistical analysis}

Statistical analyses were performed using SPSS for Windows ver. 16.0.2 (SPSS Inc., Chicago, IL, USA). Continuous data are presented as mean \pm standard deviation or as median and interquartile range according to distribution. Categorical data are presented as frequency or percentage. For dichotomous outcomes, treatment groups were compared by means of the $\chi^{2}$ test. Independent $t$ test was used to compare means. The Mann-Whitney $U$ test was used for continuous, not normally distributed outcomes. Ordinal logistic regression was used to determine possible factors prognostic for the extent of adhesiolysis during CP. Linear 
Table 1 Open versus laparoscopic emergency colectomy
Data are presented as mean $\pm \mathrm{SD}$ and median [IQR]

$E C$ emergency colectomy, $B M I$ body mass index, $U C$ ulcerative colitis, IPAA ileopouch anal anastomosis, $C P$ completion proctectomy, $I H$ incisional hernia

a $\chi^{2}$ test

${ }^{\mathrm{b}}$ independent $t$ test

c Fisher's exact test (two-tailed)

d Mann-Whitney $U$ test

e $\chi^{2}$ test for trend

\begin{tabular}{|c|c|c|c|}
\hline & EC open $(n=64)$ & EC laparoscopic $(n=36)$ & $P$ value \\
\hline \multicolumn{4}{|l|}{ Baseline characteristics } \\
\hline Gender ( $\%$ male $)$ & 59.4 & 58.3 & $0.919^{\mathrm{a}}$ \\
\hline Age at colectomy (years) & $39 \pm 12.7$ & $33 \pm 10.3$ & $0.013^{\mathrm{b}}$ \\
\hline BMI $\left(\mathrm{kg} / \mathrm{m}^{2}\right)$ & $24.7 \pm 3.2$ & $22.9 \pm 4.7$ & $0.110^{\mathrm{b}}$ \\
\hline Previous midline laparotomy $[n(\%)]$ & $1(1.6)$ & $0(0)$ & $1.000^{\mathrm{b}}$ \\
\hline Duration UC until colectomy (months) & $22[3-75]$ & $37[11-80]$ & $0.329^{\mathrm{d}}$ \\
\hline \multicolumn{4}{|l|}{ Emergency colectomy } \\
\hline Conversion $[n(\%)]$ & - & $2(5.6)$ & - \\
\hline Perforation (spontaneous) $[n(\%)]$ & $9(14.1)$ & $1(2.8)$ & $0.082^{\mathrm{c}}$ \\
\hline Overall morbidity $<30$ days $[n(\%)]$ & $24(37.5)$ & $6(16.7)$ & $0.025^{\mathrm{a}}$ \\
\hline Major morbidity $<30$ days $[n(\%)]$ & $13(20.3)$ & $2(5.6)$ & $0.045^{\mathrm{c}}$ \\
\hline Reoperation $<30$ days post op $[n(\%)]$ & $10(15.6)$ & $2(5.6)$ & $0.200^{\mathrm{c}}$ \\
\hline \multicolumn{4}{|l|}{ Completion proctectomy with IPAA } \\
\hline Adhesiolysis (overall) & - & - & $<0.001^{\mathrm{e}}$ \\
\hline None & $11(17.2)$ & $23(63.9)$ & $<0.001^{\mathrm{a}}$ \\
\hline Limited & $22(34.4)$ & $11(30.6)$ & $0.658^{\mathrm{a}}$ \\
\hline Extensive & $30(46.9)$ & $2(5.6)$ & $<0.001^{\mathrm{a}}$ \\
\hline Operating time $(\min )$ & $157[128-183]$ & $163[132-180]$ & $0.997^{\mathrm{d}}$ \\
\hline $\mathrm{IH}$ correction during $\mathrm{CP}[n(\%)]$ & $9(14.3)$ & $0(0)$ & $0.024^{\mathrm{c}}$ \\
\hline Overall morbidity $<30$ days $[n(\%)]$ & $21(32.8)$ & $7(19.4)$ & $0.176^{\mathrm{a}}$ \\
\hline Reoperation $<30$ days post op [ $n(\%)]$ & $3(4.7)$ & $2(5.6)$ & $1.000^{\mathrm{c}}$ \\
\hline Readmission $<30$ days post op [ $n(\%)]$ & $7(10.9)$ & $1(2.8)$ & $0.253^{\mathrm{c}}$ \\
\hline Mortality $<30$ days $[n(\%)]$ & $0(0)$ & $0(0)$ & - \\
\hline Total postoperative hospital stay (days) & $10[8-13]$ & $9[8-11]$ & $0.052^{\mathrm{d}}$ \\
\hline Interval acute colectomy and CP (months) & $12[7-21]$ & $6[5-14]$ & $0.001^{\mathrm{d}}$ \\
\hline
\end{tabular}

regression was used to determine possible factors prognostic for a longer operating time. Because the outcome variable "operating time" was not normally distributed, these data were log-transformed upon entry into linear regression. Considering the number of variables and sound

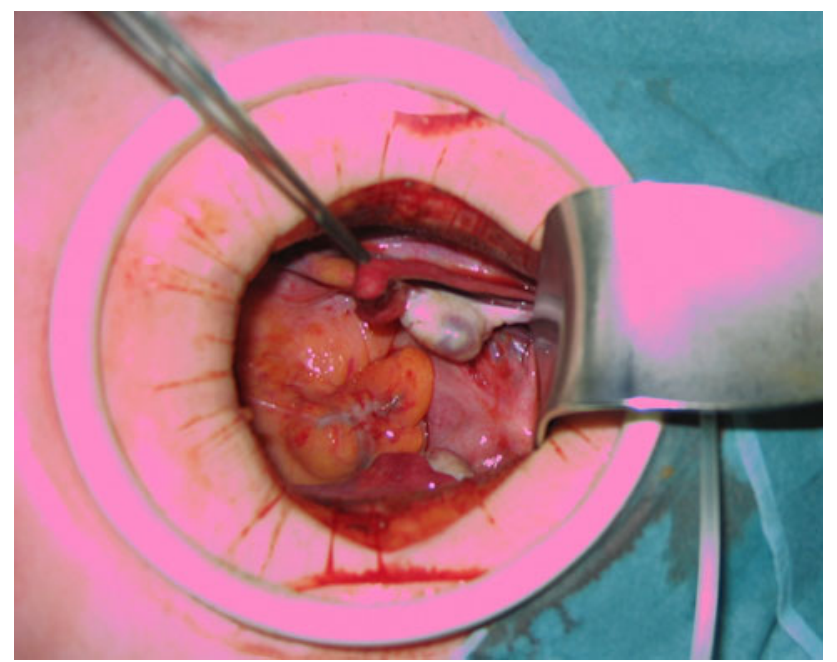

Fig. 1 Completion proctectomy after laparoscopic colectomy: no adhesions present clinical grounds for inclusion, all possible prognostic factors were considered in further multivariable analysis. A $P$ value $<0.05$ was considered to be statistically significant.

\section{Results}

In the study period, 100 patients underwent $\mathrm{CP}$ with IPAA at the AMC, The Netherlands. Emergency colectomy had been carried out laparoscopically in 36 patients: 30 in the AMC and 6 in a referring hospital. Sixty-four patients had an open emergency colectomy: 22 in the AMC and 42 in a referring hospital.

\section{Open versus laparoscopic colectomy (Table 1)}

Baseline characteristics were similar, apart from patient age at colectomy, which was higher for the group that underwent open colectomy ( $39 \pm 13$ vs. $33 \pm 10$ years, $P=0.013$ ). Adhesiolysis during $\mathrm{CP}$ was performed significantly less often and less extensively after a laparoscopic emergency colectomy compared to an open approach $(P<0.001)$ Fig. 1 . Also, incisional hernia correction during $\mathrm{CP}$ was performed significantly more often 
after open colectomy (14 vs. $0 \%, P=0.024)$. CP was performed within a shorter period of time after laparoscopic colectomy (median 12 vs. 6 months, $P=0.001$ ).

\section{Colectomy at referring hospital versus AMC (Table 2)}

Adhesiolysis during CP and IPAA occurred significantly less often and was less extensively carried out in the group whose initial operation was at the AMC $(P=0.004)$. Median operating time and rate of incisional hernia correction were similar for both groups. Median interval to $\mathrm{CP}$ for the referred versus AMC groups was 20 and 8 months, respectively $(P<0.001)$.

\section{Multivariable analysis}

Six possible factors that could influence the extent of adhesiolysis during $\mathrm{CP}$ were analyzed using univariable and subsequent multivariable ordinal logistic regression: "open colectomy," "colectomy in referring hospital," "previous midline laparotomy," "reoperation within 30 days after initial colectomy," "spontaneous perforation," and "major complications after emergency colectomy." Both "open colectomy" and "colectomy in referring hospital" seemed significant predictors in the univariable analysis ( $P<0.001$ and $P=0.003$, respectively). However, multivariable regression analysis showed that the only independent predictive factor of an increase in the extent of adhesiolysis during CP was "open colectomy" $[P<0.001$, OR 8.70 (95\% CI: 3.29-22.98)]. The remaining variables were not independently predictive of the need for adhesiolysis during $\mathrm{CP}$.

\section{Operating time}

Univariable and subsequent multivariable linear regression analyses were done to determine independent predictive factors for operating time of CP. Auxiliary analyses on logtransformed operating times showed similar results; for ease of interpretation, the results from the analyses on nontransformed data are shown (Table 3). "Extent of adhesiolysis" was an independent predictive factor for a longer operating time of the $\mathrm{CP}$ and IPAA (overall test degrees of freedom $=2 ; P<0.001)$, i.e., limited adhesiolysis adds $18(0-36)$ min and extensive adhesiolysis adds 55 (35-75) $\mathrm{min}$ to the operating time. Moreover, operating time of the CP was significantly longer if the initial colectomy had been performed at a referring hospital
Table 2 Emergency colectomy in referring hospital versus AMC

\footnotetext{
Data are presented as mean $\pm \mathrm{SD}$ and median [IQR]

$\mathrm{EC}=$ emergency colectomy;

$\mathrm{BMI}=$ body mass index;

$\mathrm{UC}=$ ulcerative colitis;

IPAA = ileopouch anal anastomosis; $\mathrm{CP}=$ completion proctectomy; $\mathrm{IH}=$ incisional hernia

a $\chi^{2}$ test

b independent $t$ test

c Fisher's exact test (two-tailed)

d Mann-Whitney $U$ test

e $\chi^{2}$ test for trend
}

\begin{tabular}{|c|c|c|c|}
\hline & EC referring center $(n=48)$ & $\operatorname{EC~} \operatorname{AMC}(n=52)$ & $P$ value \\
\hline \multicolumn{4}{|l|}{ Baseline characteristics } \\
\hline Gender (\% male) & 58.3 & 59.6 & $0.896^{\mathrm{a}}$ \\
\hline Age at colectomy (years) & $39 \pm 12.4$ & $36 \pm 13.2$ & $0.345^{\mathrm{b}}$ \\
\hline BMI $\left(\mathrm{kg} / \mathrm{m}^{2}\right)$ & $24.5 \pm 3.6$ & $23.7 \pm 4.5$ & $0.108^{\mathrm{b}}$ \\
\hline Previous midline laparotomy $[n(\%)]$ & $0(-)$ & $1(1.9)$ & $1.000^{\mathrm{c}}$ \\
\hline Duration UC until colectomy (months) & $25.8[3-73]$ & 38 [12-94] & $0.284^{\mathrm{d}}$ \\
\hline \multicolumn{4}{|l|}{ Emergency colectomy } \\
\hline Conversion $[n(\%)]$ & $0(0)$ & $2(3.8)$ & - \\
\hline Perforation (spontaneous) $[n(\%)]$ & $8(21.1)$ & $2(3.8)$ & $0.012^{\mathrm{c}}$ \\
\hline Overall morbidity $<30$ days $[n(\%)]$ & $18(37.5)$ & $12(23.1)$ & $0.041^{\mathrm{a}}$ \\
\hline Major morbidity $<30$ days $[n(\%)]$ & $9(18.8)$ & $6(11.5)$ & $0.211^{\mathrm{c}}$ \\
\hline Reoperation $<30$ days postop $[n(\%)]$ & $7(14.6)$ & $5(9.6)$ & $0.308^{\mathrm{c}}$ \\
\hline \multicolumn{4}{|l|}{ Completion proctectomy with IPAA } \\
\hline Adhesiolysis (overall) & - & - & $0.004^{\mathrm{e}}$ \\
\hline None & $10(20.8)$ & $24(46.2)$ & $0.009^{\mathrm{a}}$ \\
\hline Limited & $16(33.3)$ & $17(32.7)$ & $0.887^{\mathrm{a}}$ \\
\hline Extensive & $21(43.8)$ & $11(21.2)$ & $0.012^{\mathrm{a}}$ \\
\hline Operating time $(\mathrm{min})$ & $169[136-190]$ & $153[128-175]$ & $0.076^{\mathrm{d}}$ \\
\hline $\mathrm{IH}$ correction during $\mathrm{CP}[n(\%)]$ & $5(10.6)$ & $4(7.7)$ & $0.611^{\mathrm{c}}$ \\
\hline Overall morbidity $<30$ days $[n(\%)]$ & $16(34.0)$ & $12(23.1)$ & $0.226^{\mathrm{a}}$ \\
\hline Reoperation $<30$ days postop $[n(\%)]$ & $4(8.3)$ & $1(1.9)$ & $0.192^{\mathrm{c}}$ \\
\hline Readmission $<30$ days postop $[n(\%)]$ & $5(10.4)$ & $3(5.8)$ & $0.475^{\mathrm{c}}$ \\
\hline Mortality $<30$ days $[n(\%)]$ & $0(0)$ & $0(0)$ & - \\
\hline Total postoperative hospital stay (days) & $9[7-15]$ & $10[9-12]$ & $0.800^{\mathrm{d}}$ \\
\hline Interval acute colectomy and $\mathrm{CP}$ (months) & 20 [11-27] & $8[6-12]$ & $<0.001^{\mathrm{d}}$ \\
\hline
\end{tabular}


Table 3 Linear regression for operating time of completion proctectomy

\begin{abstract}
$C P$ completion proctectomy with ileopouch anal anastomosis, $\mathrm{CI}$ confidence interval
\end{abstract}

\begin{tabular}{lccl}
\hline Variable & $\begin{array}{l}\text { Univariable } \\
P \text { value }\end{array}$ & $\begin{array}{l}\text { Multivariable } \\
P \text { value }\end{array}$ & B (95\% CI) \\
\hline Emergency colectomy in referring hospital & 0.018 & 0.013 & $19.9(4.3-35.5)$ \\
Major complications of emergency colectomy & 0.959 & 0.366 & - \\
CP performed through Pfannenstiel incision & 0.691 & $<0.001$ & $35.1(16.0-54.3)$ \\
Extent of adhesiolysis during CP (overall test) & $<0.001$ & $<0.001$ & - \\
None & - & - & - \\
Limited & 0.204 & 0.046 & $18.2(0.3-36.1)$ \\
Extensive & $<0.001$ & $<0.001$ & $55.0(35.0-75.0)$ \\
Loop ileostomy during CP & 0.185 & 0.069 & - \\
Incisional hernia correction during CP & 0.188 & 0.082 & - \\
\hline
\end{tabular}

$(P=0.013)$ and if the $\mathrm{CP}$ was performed through a Pfannenstiel incision instead of a midline incision $(P<0.001)$.

\section{Discussion}

Data from this study suggest that adhesiolysis is performed less often and less extensively after laparoscopic emergency colectomy. Multivariable analysis showed that the difference in adhesiolysis during $\mathrm{CP}$ between referred patients and those initially treated at the AMC could be attributed to the fact that emergency colectomy in the academic setting was more often performed laparoscopically. Extent of adhesiolysis, emergency colectomy at a referring hospital, and performing CP through a Pfannenstiel incision were significant independent predictive factors for a longer operating time of the CP. However, operating time of the CP did not differ between the laparoscopic and open groups initially, even though significantly more adhesiolysis had to be carried out after an open emergency colectomy (Table 1). This can be attributed to the fact that $\mathrm{CP}$ performed through a Pfannenstiel incision takes more time because it is more complex, therefore compensating for the initial gain in operating time by not having to perform adhesiolysis. Laparoscopic colectomy led to a lower rate of incisional hernia corrections during CP. Morbidity, mortality, and postoperative length of stay of the CP were not influenced by the surgical approach or the hospital setting of the colectomy.

Adhesions are the result of a disturbed equilibrium between fibrinogenesis and fibrinolysis. The peritoneal trauma caused by surgery dramatically decreases fibrinolytic activity [12]. Total colectomy leaves a large retroperitoneal wound surface apart from the incision used for approach and extraction. Laparoscopic surgery reduces this trauma in several ways: smaller peritoneal incision size, a closed and more humid environment, fewer foreign bodies, and less tissue trauma and hemorrhage, thereby contributing to fibrinolytic activity and thus reducing adhesions [13].

The interval to $\mathrm{CP}$ was shorter after laparoscopic surgery and after colectomy in the AMC. A similar finding has been described by Chung et al. [14]. CP within 3 months is associated with a higher risk of intraoperative complications and a higher rate of fistula formation [4]. Perhaps patients who underwent open colectomy were in worse condition than patients who underwent laparoscopic colectomy, e.g., the patients with spontaneous perforation. Another explanation could be found in referral patterns; gastroenterologists and surgeons in referring hospitals are less familiar with pouch surgery which may cause delay in referring the patient for $\mathrm{CP}$ with IPAA.

The results of this study corroborate the findings of earlier clinical studies such as that of Indar et al. [7] in which adhesions were prospectively scored during surgery following earlier colorectal procedures. In a systematic review, Gutt et al. [13] also found a reduction in adhesion formation after laparoscopic surgery in clinical and mostly experimental studies published up to 2004 .

The major limitation of this retrospective study is the way adhesiolysis was scored. The gastrointestinal surgeons reported routinely the presence of adhesions and the necessity and extent of adhesiolysis in the operation notes. Ideally, adhesions would have been scored prospectively by a validated scoring system, like the one used for gynecological surgery [15]. Such a system would reduce inter- and intraobserver variability and it would distinguish the sites of adhesion formation, e.g., abdominal wall or ovarian tubes. However, these validated scoring lists do not account for the extent of small-bowel adhesions, which are largely responsible for prolonged operating times and risk of additional morbidity. Scoring the extent of adhesiolysis probably better reflects the consequences of the presence of adhesions. Patients with a known spontaneous perforation at the time of the initial colectomy all had a midline 
laparotomy. Abdominal contamination and peritonitis can also cause adhesion formation, therefore constituting a potential bias, even though the percentage of perforations was not significantly higher in the group that underwent open colectomy.

Adhesiolysis can lead to serious complications, such as inadvertent enterotomy during reopening of the abdomen after previous abdominal surgery. Bleeding, damage to the surrounding organs, and conversion to laparotomy are also more likely to occur. Besides, earlier studies confirm that adhesiolysis increases operating time: an extra $20 \mathrm{~min}$ on average [8]. Therefore, the reduction in need for adhesiolysis is an important argument for laparoscopic emergency colectomy. Moreover, fecundity might be better preserved with laparoscopic colectomy [7]. Nevertheless, laparoscopic surgery requires an experienced surgeon, operating time is often longer, and it is more costly. It is probably not feasible for all referring hospitals to perform laparoscopic colectomies, all the more so because this emergency procedure is often done by the on-call surgeon. If laparoscopy is not possible, the emergency colectomy is preferably done by a specialized gastrointestinal surgeon, minimizing surgical trauma to the already vulnerable peritoneum in patients with ulcerative colitis.

In conclusion, laparoscopic as opposed to open emergency colectomy is associated with less need for adhesiolysis, fewer incisional hernias, and a shorter interval to completion proctectomy.

Acknowledgments The authors thank Ir. N. van Geloven for her help with the statistical analysis.

Disclosures Prof. Dr. Bemelman has received an unrestricted research grant from Ethicon Endo-Surgery Europe GmbH (part of Johnson \& Johnson). Drs. Bartels, Vlug, Henneman, Ponsioen, and Tanis have no conflicts of interest or financial ties to disclose.

Open Access This article is distributed under the terms of the Creative Commons Attribution Noncommercial License which permits any noncommercial use, distribution, and reproduction in any medium, provided the original author(s) and source are credited.

\section{References}

1. Russel MG, Dorant E, Volovics A, Brummer RJ, Pop P, Muris JW, Bos LP, Limonard CB, Stockbrugger RW (1998) High incidence of inflammatory bowel disease in The Netherlands: results of a prospective study. The South Limburg IBD Study Group. Dis Colon Rectum 41:33-40

2. Shivananda S, Lennard-Jones J, Logan R, Fear N, Price A, Carpenter L, van Blankenstein M (1996) Incidence of inflammatory bowel disease across Europe: is there a difference between north and south? Results of the European Collaborative Study on Inflammatory Bowel Disease (EC-IBD). Gut 39:690-697

3. Bach SP, Mortensen NJ (2007) Ileal pouch surgery for ulcerative colitis. World J Gastroenterol 13:3288-3300

4. Dinnewitzer AJ, Wexner SD, Baig MK, Oberwalder M, Pishori T, Weiss EG, Efron J, Nogueras JJ, Vernava AM III (2006) Timing of restorative proctectomy following subtotal colectomy in patients with inflammatory bowel disease. Colorectal Dis $8: 278-282$

5. Ahmed AU, Keus F, Heikens JT, Bemelman WA, Berdah SV, Gooszen HG, van Laarhoven CJ (2009) Open versus laparoscopic (assisted) ileo pouch anal anastomosis for ulcerative colitis and familial adenomatous polyposis. Cochrane Database Syst Rev (1):CD006267

6. Dowson HM, Bong JJ, Lovell DP, Worthington TR, Karanjia ND, Rockall TA (2008) Reduced adhesion formation following laparoscopic versus open colorectal surgery. Br J Surg 95: 909-914

7. Indar AA, Efron JE, Young-Fadok TM (2009) Laparoscopic ileal pouch-anal anastomosis reduces abdominal and pelvic adhesions. Surg Endosc 23:174-177

8. van Gool H (2007) Consequences and complications of peritoneal adhesions. Colorectal Dis 9(Suppl 2):25-34

9. Sileri P, Sthory R, McVeigh E, Child T, Cunningham C, Mortensen NJ, Lindsey I (2008) Adhesions are common and costly after open pouch surgery. J Gastrointest Surg 12:1239-1245

10. Taylor GW, Jayne DG, Brown SR, Thorpe H, Brown JM, Dewberry SC, Parker MC, Guillou PJ (2010) Adhesions and incisional hernias following laparoscopic versus open surgery for colorectal cancer in the CLASICC trial. Br J Surg 97:70-78

11. Mudge M, Hughes LE (1985) Incisional hernia: a 10 year prospective study of incidence and attitudes. Br J Surg 72:70-71

12. Schippers E, Tittel A, Ottinger A, Schumpelick V (1998) Laparoscopy versus laparotomy: comparison of adhesion-formation after bowel resection in a canine model. Dig Surg 15:145-147

13. Gutt CN, Oniu T, Schemmer P, Mehrabi A, Buchler MW (2004) Fewer adhesions induced by laparoscopic surgery? Surg Endosc 18:898-906

14. Chung TP, Fleshman JW, Birnbaum EH, Hunt SR, Dietz DW, Read TE, Mutch MG (2009) Laparoscopic vs. open total abdominal colectomy for severe colitis: impact on recovery and subsequent completion restorative proctectomy. Dis Colon Rectum 52:4-10

15. Adhesion Scoring Group (1994) Improvement of interobserver reproducibility of adhesion scoring systems. Fertil Steril 62: 984-988 